\title{
Spherical tokamaks with a high current carrying plasma center column
}

Cite as: Phys. Plasmas 25, 122513 (2018); https://doi.org/10.1063/1.5066221

Submitted: 12 October 2018 . Accepted: 02 December 2018 . Published Online: 19 December 2018

L. G. Lampugnani (D), P. L. Garcia-Martinez, and R. Farengo
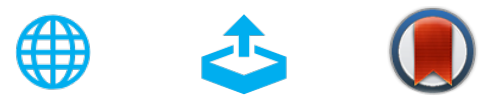

View Online

Export Citation

\section{ARTICLES YOU MAY BE INTERESTED IN}

Fast evaluation of the current driven by electron cyclotron waves for reactor studies Physics of Plasmas 25, 122501 (2018); https://doi.org/10.1063/1.5050345

Neoclassical quasilinear theory and universal collision frequency landscape in wave-particle interaction in tokamaks

Physics of Plasmas 25, 122502 (2018); https://doi.org/10.1063/1.5046112

Hybrid simulations of fishbone instabilities and Alfvén eigenmodes in DIII-D tokamak

Physics of Plasmas 25, 122504 (2018); https://doi.org/10.1063/1.5064647 


\title{
Spherical tokamaks with a high current carrying plasma center column
}

\author{
L. G. Lampugnani, ${ }^{1,2,3, a)}$ P. L. Garcia-Martinez, ${ }^{2,3,4}$ and R. Farengo ${ }^{1,3}$ \\ ${ }^{1}$ Instituto Balseiro, UNCuyo, Av. Bustillo 9500, 8400 Bariloche, Argentina \\ ${ }^{2}$ CCT Patagonia Norte (CONICET), 8400 Bariloche, Argentina \\ ${ }^{3}$ Centro Atómico Bariloche (CNEA), Av. Bustillo 9500, 8400 Bariloche, Argentina \\ ${ }^{4} U N R N-S e d e$ Andina, 8400 Bariloche, Argentina
}

(Received 12 October 2018; accepted 2 December 2018; published online 19 December 2018)

\begin{abstract}
Spherical tokamaks (STs) have many advantages from the perspective of a fusion reactor. A further improvement would be to replace the center post by a plasma center column (PCC). In this case, biased electrodes could be used to drive current along the PCC and produce the toroidal magnetic field. Moreover, the magnetic helicity injected (HI) by the PCC can be used to form and sustain the configuration, via magnetic relaxation. The magnetic structure and stability of these so-called STPCC configurations are studied in detail. In particular, it is shown that stable equilibria with tokamak-like safety factor $(q)$ profiles can be obtained in the regime of high PCC current and moderate poloidal flux amplification. Using numerical simulations, the feasibility of forming and sustaining ST-PCC configurations via HI is demonstrated. The sustainment in this case involves a significant level of fluctuations and is shown to occur at a marginally stable configuration having a $q=1$ surface in the ST. This behavior is in close analogy to that of spheromaks sustained by a coaxial plasma gun but presents two major differences. First, the current density in the open flux region (PCC) is significantly larger. Second, the mean current density gradient in the ST has the opposite sign, leading to $q$ profiles with regular magnetic shear (i.e., $q$ increases from the magnetic axis to the separatrix, as in tokamaks). When helicity injection is switched off, the fluctuations decay and nested, closed magnetic surfaces appear. This opens the possibility of using HI to form the ST-PCC and a combination of auxiliary current drive (neutral beams and/or RF) and high bootstrap current to sustain a fluctuation free configuration. Published by AIP Publishing.

https://doi.org/10.1063/1.5066221
\end{abstract}

\section{INTRODUCTION}

Spherical torus or tokamak plasmas (STs) are toroidal confinement configurations with a very small aspect ratio. It has been demonstrated that they have a number of attractive physical features, including very high plasma $\beta$, naturally high plasma elongation, and high non-inductive current fraction. ${ }^{1,2}$ From the perspective of a fusion reactor, the low aspect ratio implies the possibility of compact fusion at low field and modest cost. In conventional spherical tokamaks, the plasma torus is linked by a central post containing the inner part of the toroidal magnet and the ohmic transformer. Due to the very limited space left at a low aspect ratio, the design of this centerpost poses very difficult challenges. For instance, the use of superconducting toroidal field magnets, which is being incorporated in some new designs, is at the cost of an increased machine size (with respect to a cooper based one) due to the need of increased inboard neutron shielding. ${ }^{3}$

To overcome these difficulties, the center-post may be replaced by a pair of electrodes linked by open flux surfaces across the plasma center. ${ }^{4-6}$ Biasing these electrodes, a poloidal current may be driven along the open flux surfaces to produce the toroidal field. With this approach, configurations with many features in common with spherical tokamaks could be obtained inside simply connected chambers. An early

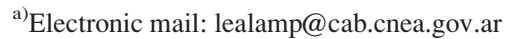

configuration of this type is the flux-core spheromak (FCS). ${ }^{7}$ A more advanced proposal is the PROTO-SPHERA experiment, ${ }^{4,6}$ which involves a central screw-pinch fed by carefully designed electrodes and surrounded by a spherical torus. A related configuration is the spherical tokamak with a plasma center column (ST-PCC), whose properties, relevant parameter space, and tilt stability have already been studied. ${ }^{5,8,9}$ Those studies, however, were restricted to current density profiles corresponding to fully relaxed equilibria.

Another advantage of this type of configuration is that formation occurs spontaneously, as a result of magnetic relaxation, when magnetic helicity is injected into the system. ${ }^{7}$ The required helicity injection (HI) may be provided either by the PCC alone ${ }^{4}$ or by the combined action of the PCC and a coaxial injector, ${ }^{5}$ similar to the coaxial plasma gun used for spheromak configurations. ${ }^{10,11}$ After formation, HI can also be employed as an efficient current-drive mechanism to sustain the configuration against resistive dissipation. ${ }^{7,11}$ Unfortunately, steady-state sustainment using $\mathrm{HI}$ and plasma relaxation necessarily involves magnetohydrodynamic (MHD) fluctuations that degrade confinement. ${ }^{10-12}$ Accordingly, experiments show improved confinement when relaxation fluctuations decrease either because the external driving is switched off (decay phase) ${ }^{13}$ or because some auxiliary driving is applied to reduce current density gradients. ${ }^{14}$ For this reason, alternative sustainment scenarios using auxiliary non-inductive current drive 
(CD) systems, such as beam injection or RF have also been proposed in this context. ${ }^{5,15}$

In this work, the analysis of ST-PCC equilibria ${ }^{5,9}$ is extended to consider arbitrary current density profiles (Sec. II). The magnetic structure of these equilibria is computed in the two dimensional parameter space defined by the flux amplification factor and the current along the PCC. Then, the stability and dynamics of relaxation are investigated by solving the non-linear time dependent MHD equations in three spatial dimensions (Sec. III). Stable equilibria with high current along the PCC and tokamak-like safety factor profiles in the ST are identified. These equilibria are of particular interest for sustainment scenarios without the MHD activity using auxiliary $\mathrm{CD}$ systems combined with the expected large bootstrap current. The stability boundary to the kink mode of the PCC is in good agreement with the Kruskal-Shafranov (KS) limit. ${ }^{16}$ Just above this limit, the kink mode grows but is rapidly stabilized by the ST, unless the PCC current is high enough to trigger relaxation. Finally, the formation and sustainment of ST-PCC configurations using $\mathrm{HI}$ are studied using numerical simulations (Sec. IV). The evolution of the magnetic flux surfaces during the decay phase is presented, showing how the quality of these surfaces is improved after HI is switched off. The main results and conclusions are summarized in Sec. V.

\section{ST-PCC EQUILIBRIA}

For the vast majority of magnetic configurations of interest in fusion research, the MHD force balance equation, $\mathbf{J} \times \mathbf{B}=\nabla p$, plays a central role in both ideal and transport time scales. This work is focused on the low pressure regime where magnetic forces are dominant. In the limit of negligible pressure force, or zero beta, the equilibrium condition is satisfied whenever the magnetic field is parallel to the current density, i.e.,

$$
\nabla \times \mathbf{B}=\Lambda(\mathbf{r}) \mathbf{B}
$$

where $\Lambda$ is some scalar function of space and all quantities are normalized using suitable scales, as described in Sec. II B.

\section{A. Force-free Grad-Shafranov equation}

In two-dimensional axisymmetric equilibria, the variables are restricted to the poloidal plane $(r, z)$ and the toroidal coordinate $\phi$ is ignorable. In such a case, the poloidal flux $\psi$ acts as a stream function for the poloidal magnetic field, and the condition (1) may be expressed as the force-free GradShafranov equation ${ }^{17}$

$$
\Delta^{*} \psi+\Lambda(\psi) \int_{0}^{\psi} \Lambda\left(\psi^{\prime}\right) d \psi^{\prime}=0,
$$

where $\Delta^{*}=\left(\partial^{2} / \partial r^{2}\right)-(1 / r)(\partial / \partial r)+\left(\partial^{2} / \partial z^{2}\right)$. The poloidal magnetic field is computed from $\psi$ as

$$
\mathbf{B}_{\mathrm{pol}}=\nabla \times\left(\frac{\psi}{2 \pi r} \hat{\phi}\right)
$$

The Grad-Shafranov equation (2) is commonly written in terms of the toroidal field function $F(\psi)=r B_{\phi}$. However, we note that

$$
2 \pi r B_{\phi}=\int_{\mathcal{C}(r, z)} J_{z} d S=\int_{0}^{r} \Lambda \underbrace{B_{z} 2 \pi r^{\prime} d r^{\prime}}_{d \psi^{\prime}}=\int_{0}^{\psi} \Lambda\left(\psi^{\prime}\right) d \psi^{\prime},
$$

where $\mathcal{C}(r, z)$ is the circle of radius $r$ centered at vertical position $z$, and we have used Ampère's law, the $z$ component of Eq. (1), and the definition of $\psi$, respectively. This can be rewritten as

$$
F(\psi)=\frac{1}{2 \pi} \int_{0}^{\psi} \Lambda\left(\psi^{\prime}\right) d \psi^{\prime},
$$

implying that $\Lambda$, likewise $F$, is a function of $\psi$ alone under the MHD equilibrium condition. This justifies the use of $\Lambda(\psi)$ in Eq. (2) instead of $\Lambda(\mathbf{r})$. Moreover, Eq. (4) allows us to obtain the toroidal field directly from the $\Lambda(\psi)$ profile.

In the first part of this paper, ST-PCC configurations are studied by solving Eq. (2) and analyzing their magnetic structure. To solve Eq. (2), appropriate boundary conditions must be imposed (see Sec. II B), and the arbitrary $\Lambda(\psi)$ profile must be specified. Dotting Eq. (1) with $\mathbf{B}$ yields

$$
\Lambda=\frac{\mathbf{J} \cdot \mathbf{B}}{B^{2}},
$$

i.e., $\Lambda(\psi)$ determines the current profile of the configuration. The profiles employed are described in Secs. II C and II D.

\section{B. Boundary conditions and normalization}

In order to obtain the magnetic configuration, the MHD equilibrium problem must be defined by prescribing the boundary conditions for the unknown $\psi$ and the current profile $\Lambda(\psi)$. With this information, Eq. (2) can be solved using a standard numerical approach suitable for non-linear partial differential equations.

The plasma is contained in a cylindrical chamber (see Fig. 3). The domain of $\psi(r, z)$ is the rectangle $[0, a] \times[-h / 2, h / 2]$, where $a$ is the chamber radius and $h$ its height. In this work, $a$ is used as the reference length scale, and all the configurations considered have elongation $h / a=1$. Thus, the domain in which Eq. (2) is to be solved is simply the unit square $(r, z) \in[0,1] \times[-1 / 2,1 / 2]$ (see Fig. 1).

Dirichlet boundary conditions are imposed on $\psi$. At $r=0$, $\psi=0$ due to symmetry (not a physical boundary). The other sides of the square represent the actual chamber walls, which are divided into electrodes (varying $\psi$ ) and perfectly conducting walls (constant $\psi$ ). In the central electrodes, $\psi$ is increased from zero at $r=0$ to $\Psi_{0}$ at the electrode radius $r_{e}$, at the two caps of the cylinder, i.e., $z= \pm 1 / 2$. The field profile at the electrodes is $B_{z}^{\text {elec }}(r)=B_{0}\left[2\left(r / r_{e}\right)^{3}-3\left(r / r_{e}\right)^{2}+1\right]$, for $r<r_{e}$, and the electrode radius is $r_{e}=a / 2$. The poloidal flux profile obtained by integrating this magnetic field is shown in Fig. 1(a) by the black lines at $z= \pm 1 / 2$. In the rest of the boundary, $\psi$ is constant and equal to $\Psi_{0}$ (and $\Psi_{0}=1$ as 

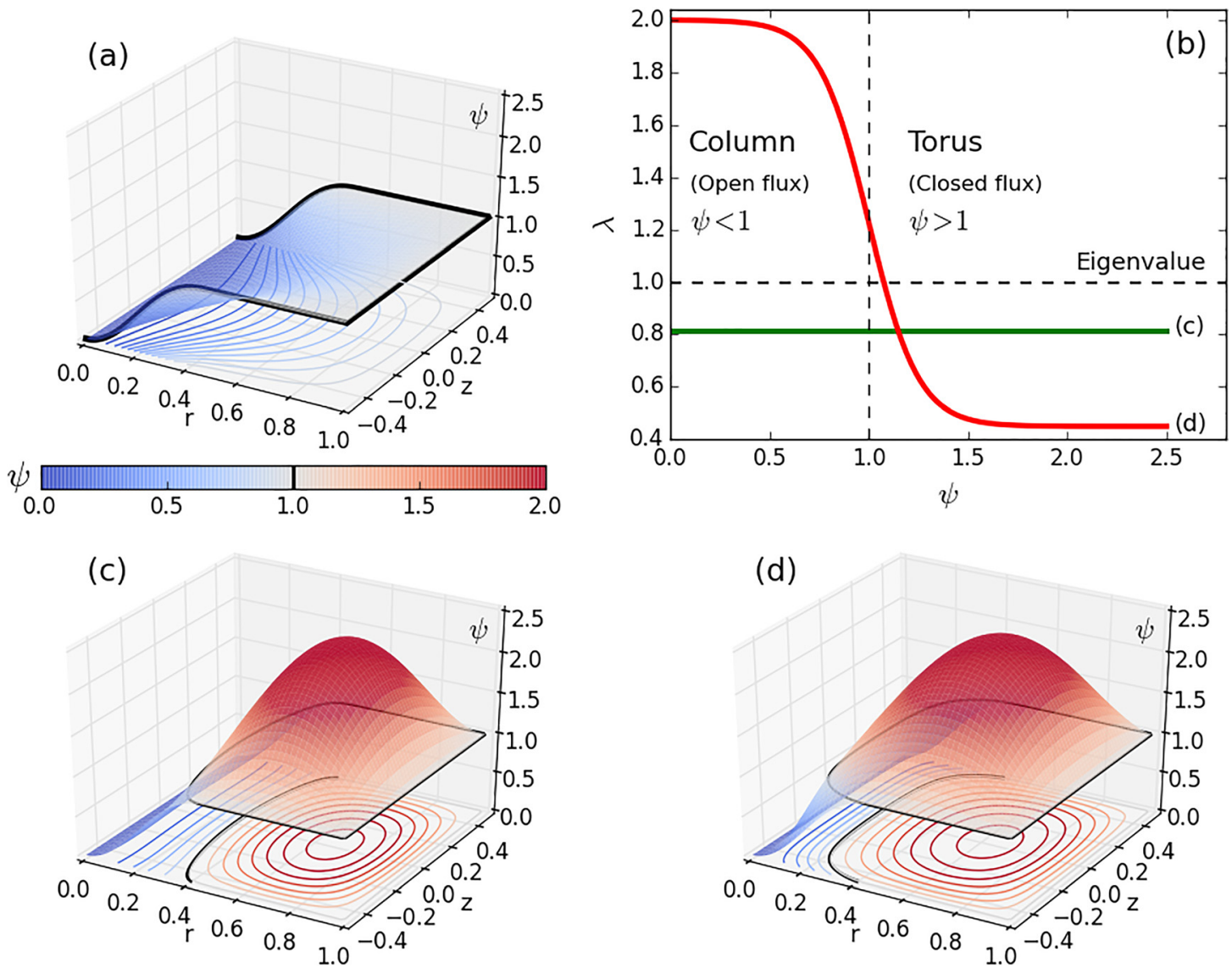

FIG. 1. (a) Vacuum solution $(\Lambda=0)$ of Eq. (2). (b) $\lambda(\psi)$ profiles for a relaxed state (green) and a configuration with high current in the plasma column (red). The solutions of Eq. (2) using these profiles are shown in panels (c) and (d), respectively.

explained below). The vacuum solution (i.e., current-free case $\Lambda=0$ ) of Eq. (2) using these boundary conditions is shown in Fig. 1(a). The same boundary conditions are applied to all the configurations presented in this paper.

Note that the magnetic flux intercepted by the electrodes is equal to one in Fig. 1(a). This is because $\Psi_{0}$ is chosen as the remaining normalizing scale (in addition to $a$ ). Using the expression for $B_{z}^{\text {elec }}(r)$, the magnetic field scale turns out to be $B_{0}=(10 / 3 \pi) \Psi_{0} / r_{e}^{2} \approx \Psi_{0} / r_{e}^{2}$. The current density scale is $J_{0}=B_{0} / a \mu_{0}$. All the quantities involved in the equilibrium problem are normalized using these scales.

\section{Relaxed states}

When an electrostatic bias is applied to the electrodes, current starts to flow along the open field lines (i.e., those lines intercepted by the electrodes). This effect is accounted for by the $\Lambda(\psi)$ profile, which must be specified to completely define the equilibrium problem.

From a mathematical point of view, the simplest option is to assume a uniform profile. For uniform $\Lambda$, Eq. (2) becomes

$$
\left(\Delta^{*}+\Lambda^{2}\right) \psi=0
$$

which is similar to the Helmholtz equation, where the GradShafranov operator replaces the Laplacian. When homogeneous boundary conditions are applied, one obtains an eigenvalue problem with non-trivial solutions only for a discrete set of eigenvalues $\Lambda_{i}$.
The first eigenfunction (corresponding to $\Lambda_{1}$ ) represents the spheromak configuration. In terms of plasma relaxation, ${ }^{7}$ this is a minimum energy state for a given amount of magnetic helicity, i.e., a relaxed state. In particular, for a cylinder $\Lambda_{1}^{2}=\left(x_{11} / a\right)^{2}+(\pi / h)^{2}$, where $x_{11}$ is the first zero of the $J_{1}$ Bessel function. ${ }^{7}$ Note that the eigenvalue problem involves homogeneous boundary conditions and thus, strictly speaking, it is limited to isolated configurations (there are no electrodes).

Relaxation theory can be extended to configurations including external (or open) flux. ${ }^{7,18}$ In this case, the uniform $\Lambda$ solution again represents a robust minimum-energy state towards which the plasma will evolve in the presence of the adequate level of fluctuations. When subjected to inhomogeneous boundary conditions, Eq. (6) has a solution for any $\Lambda$ value except for the eigenvalues, where the system behaves resonantly, i.e., $|\psi| \rightarrow \infty$ as $\Lambda \rightarrow \Lambda_{i}$. This implies that $\Lambda$ acts as a free parameter that controls the energy and helicity contents of the system.

Because of this resonant behavior, the eigenvalues also play an important role in configurations with open flux. For this reason, we take $\Lambda_{1}$ as a reference value to rescale $\Lambda$ and specify the current profile in terms of $\lambda$, defined as

$$
\lambda(\psi)=\frac{\Lambda(\psi)}{\Lambda_{1}} .
$$

The solution to Eq. (2) imposing the uniform profile $\lambda$ $\approx 0.805$ is shown in Fig. 1(c). The presence of field-aligned currents produces a region with $\psi>1$, i.e., poloidal flux 
values exceeding the external bias or flux amplification. Note that the region with $\psi<1$ is composed of open flux surfaces (those intercepting the electrodes) while the region with $\psi>1$ is formed by a collection of nested toroidal flux surfaces (or closed flux). As indicated in Fig. 1(b), the former region is the plasma central column (PCC), and the latter corresponds to the spherical tokamak (ST) or torus. The contour $\psi=1$ (shown in black) is the separatrix.

\section{Equilibria with non-uniform current profiles}

Uniform $\lambda$ configurations provide a reasonable approximation to the actual fields while involving simple linear equations. For these reasons, they have been used to introduce and study many features of ST-PCC plasmas in the past. ${ }^{5,9}$ However, the $\lambda$ profiles observed during sustainment are not uniform because different current drive mechanisms act on the open and closed flux regions. ${ }^{19}$ In this sense, the model can be improved by using a tanh $\lambda$ profile such as ${ }^{20,21}$

$$
\lambda(\psi)=\lambda_{t}+\frac{\lambda_{c}-\lambda_{t}}{2}\left[1+\tanh \left(\frac{1-\psi}{\delta}\right)\right],
$$

where $\lambda_{c}$ is the value at the central column (imposed by the electrodes), $\lambda_{t}$ is the value at the torus, proportional to the current driven by other sources (e.g., relaxation, RF neutral beams, and bootstrap), and $\delta$ is the width of the transition layer. The resulting profile for $\lambda_{c}=2$ and $\lambda_{t}=0.449$ is shown in Fig. 1(b) (red line). All the equilibria presented in this work were all computed using $\delta=0.25$; thus, in what follows, the pair of values $\left(\lambda_{c}, \lambda_{t}\right)$ completely determines the equilibrium configuration.

For non-uniform $\lambda(\psi)$ profiles, Eq. (2) becomes nonlinear, and there are no general results regarding the existence and uniqueness of the solution. In practice, the standard Newton-Raphson method converges in a few iterations for the tanh profiles considered, when the uniform $\lambda$ solution is provided as initial guess. The solution obtained for the nonuniform profile of Fig. 1(b) is shown in Fig. 1(d). Note that this configuration encloses the same amount of poloidal fluxes (open and closed) than the uniform $\lambda$ case presented in that figure. In spite of this, the compression of the plasma central column due to the high current along the open field lines can be clearly observed.

The radial profiles of the poloidal and toroidal fields at $z=0$ for the two cases are shown in Fig. 2. Although the difference between the solutions shown in Figs. 1(c) and 1(d) may seem subtle, the magnetic field profiles reveal that the magnetic structure differs significantly. The magnetic structure is further depicted by the field line plots of Fig. 3. The pinch effect, i.e., the compression of the central column, observed for the non-uniform $\lambda$ case, is also evident in these plots. Moreover, the twisting of the field lines at the central column is higher for this case, as expected due to the higher current level.

A different scenario is observed in the vicinity of the magnetic axis. In Fig. 2, the position of the magnetic axis is indicated by the zero crossing of $B_{z}$. In the non-uniform $\lambda$ case, the magnetic axis is shifted towards the center, and

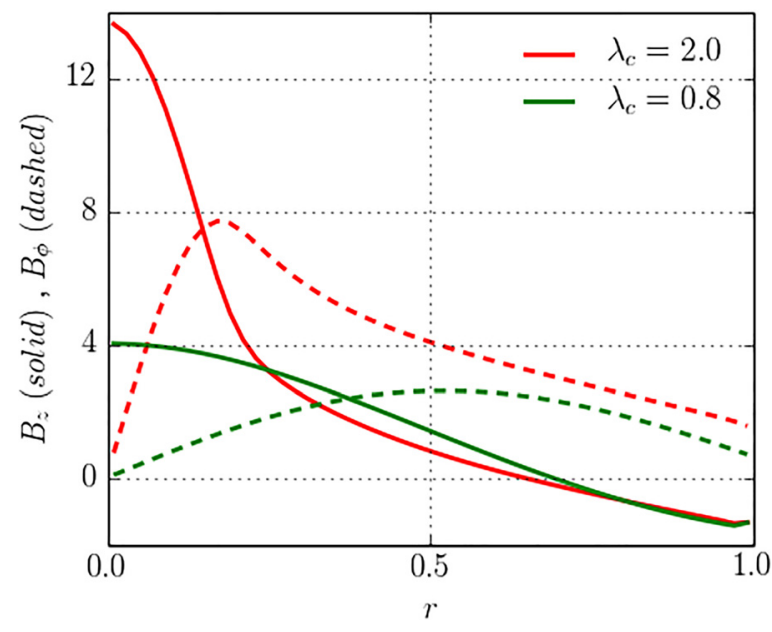

FIG. 2. Radial profiles, at $z=0$, of the poloidal $\left(B_{z}\right.$, solid) and toroidal $\left(B_{\phi}\right.$, dashed) fields for the two equilibria shown in Figs. 1(c) and 1(d).

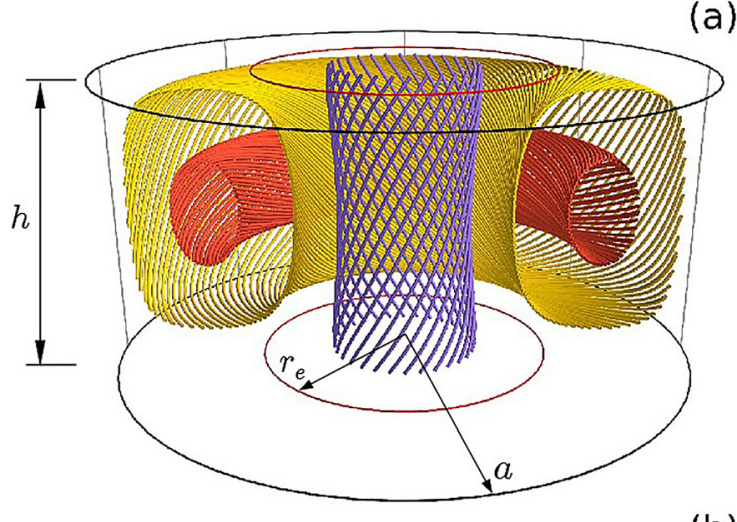

(b)

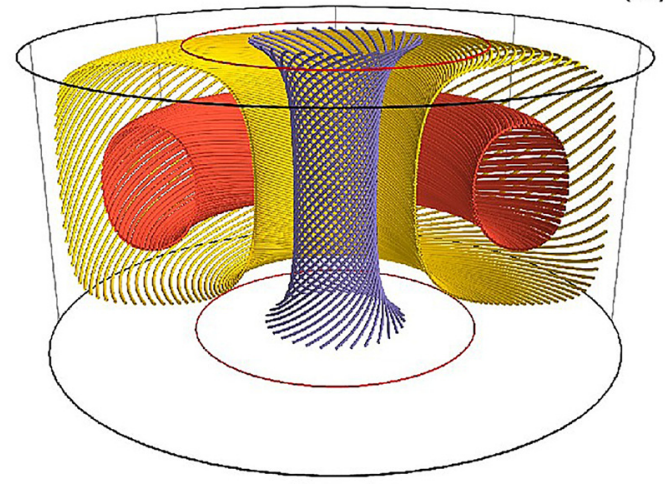

FIG. 3. Field lines of the configurations obtained for the uniform (a) and the tanh (b) $\lambda$ profiles as shown in Fig. 1.

the toroidal current in that region (proportional to the slope of $B_{z}$ ) is reduced. The closed flux surfaces are wider, compared to those of the uniform $\lambda$ case, and their field lines are less twisted (see Fig. 3).

\section{E. Amplification factor and parameter space}

The flux amplification factor, $\mathcal{A}$, defined as the ratio of the maximum poloidal flux (at the magnetic axis) to the external magnetic bias, is a conventional measure of the 
effectiveness of a current drive scheme. Using the normalization introduced in Sec. II B, it becomes

$$
\mathcal{A}=\max (\psi)
$$

The value of $\mathcal{A}$ for different configurations is shown in Fig. 4. Each black line corresponds to a fixed $\lambda_{t}$ value, and $\lambda_{c}$ is increased from $\lambda_{c}=\lambda_{t}$. Therefore, the left most values, which are connected by a blue curve, show the resonant behavior of $\mathcal{A}$ for the uniform $\lambda$ case (see Sec. II C). Note that each point in this parameter space corresponds to one particular equilibrium configuration. As an example, the points corresponding to the equilibria shown in Figs. 1(c) and $1(\mathrm{~d})$ are indicated.

When the $\lambda(\psi)$ profile is given by Eq. (8), an increase in either $\lambda_{c}$ or $\lambda_{t}$ produces an increase in $\mathcal{A}$; however, the behavior is not resonant. This is in agreement with previous studies that reported the regularization of the resonance for non-uniform $\lambda(\psi)$ profiles with a negative slope. $^{17}$

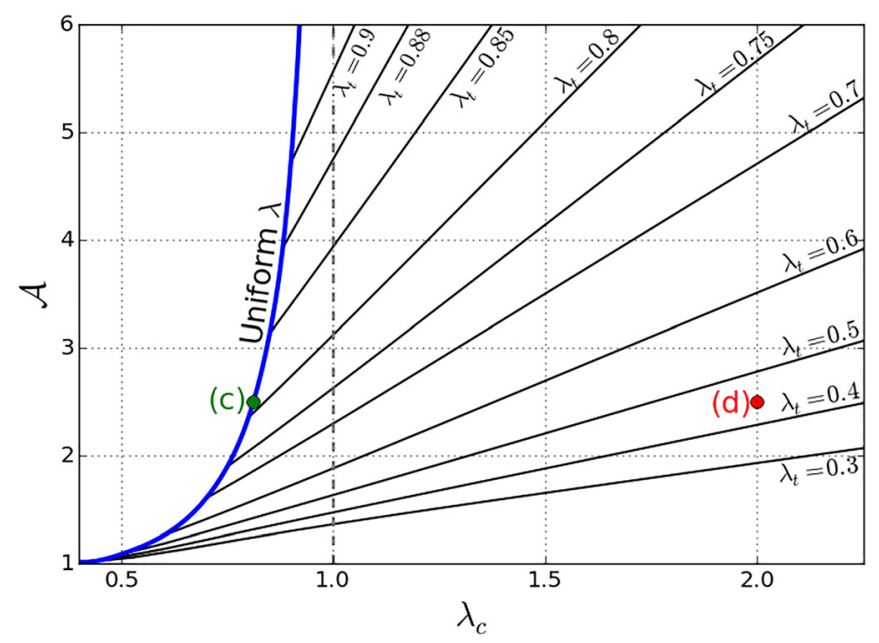

FIG. 4. Amplification factor as a function of $\lambda_{c}$ for different $\lambda_{t}$. Only cases with $\lambda_{c} \geq \lambda_{t}$ are considered. The limit $\lambda_{t}=\lambda_{c}$ corresponds to the uniform $\lambda$ case (shown in blue). The green and red points indicate the two cases shown in Figs. 1(c) and 1(d), respectively.

\section{F. Safety factor profiles}

The safety factor, $q$, is determined by the current distribution and plays a major role in the stability of the configuration. For a toroidally axisymmetric equilibrium, each field line follows a helical path and $q$ is defined as the ratio of toroidal to poloidal angle along the field line. This definition applies straightforwardly to the closed flux region. The safety factor in the torus is then computed as

$$
q_{t}=\frac{\Delta \phi}{2 \pi} \quad \text { for } \quad \psi>1,
$$

where $\Delta \phi$ is the change in the toroidal angle after one poloidal transit around the magnetic axis.

This definition cannot be applied to the central column because of the lack of periodicity in the $z$ direction. A common workaround is to consider the screw-pinch as a "straight tokamak," assuming periodicity in $z$ with period $2 \pi L$, where $L$ is the distance between the electrodes ${ }^{16}$ ( $L=h=1$ here). The safety factor in the central column is then computed as

$$
q_{c}=\frac{2 \pi}{\Delta \phi_{\text {elec }}} \quad \text { for } \quad \psi<1,
$$

where $\Delta \phi_{\text {elec }}$ is the toroidal angle each field line advances as it goes from one electrode to the other. Note that in this context, $z$ acts as the "toroidal" direction, while $\phi$ acts as the "poloidal" direction around the geometric axis.

The $\lambda(\psi)$ profiles of several cases with increasing $\lambda_{c}$ and decreasing $\mathcal{A}$ are shown in Fig. 5(a). The corresponding radial $q$ profiles, computed using Eqs. (10) and (11), are shown in Fig. 5(b). Dashed lines are used to indicate the open flux region and solid lines to indicate the closed flux region. Because of the use of different definitions, typical $q(r)$ profiles are discontinuous at the separatrix. Note that the separatrix is always at $\psi=1$ [see panel (a)] but adopts slightly different radial positions [see panel (b)]. The radial position of the separatrix is affected by the pinch effect identified in Sec. II D, but it is also affected by $\mathcal{A}$, since a larger flux content in the closed flux region may compress the central column. However, the latter effect is weaker than
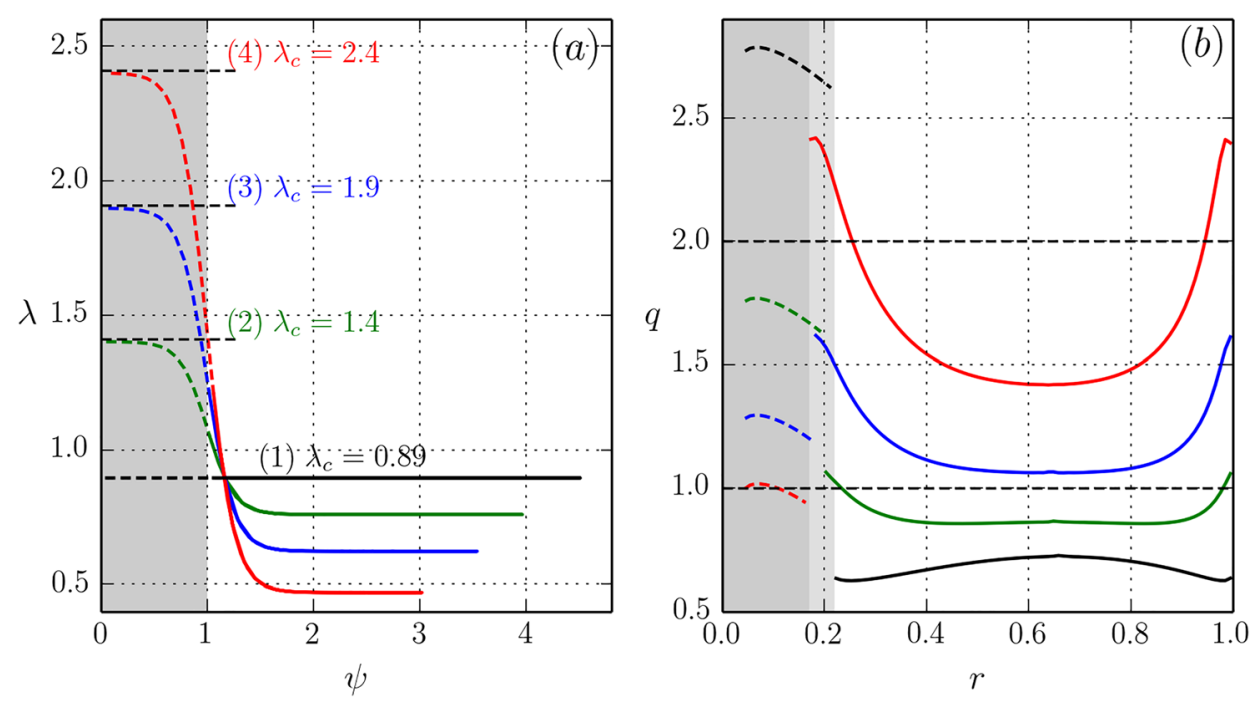

FIG. 5. $\lambda(\psi)$ profiles (a) and radial $q$ profiles (b) for several configurations with different $\lambda_{c}$ and $\mathcal{A} . q$ is computed using Eq. (11) in the PCC (gray region, dashed lines) and Eq. (10) in the torus (solid lines). 


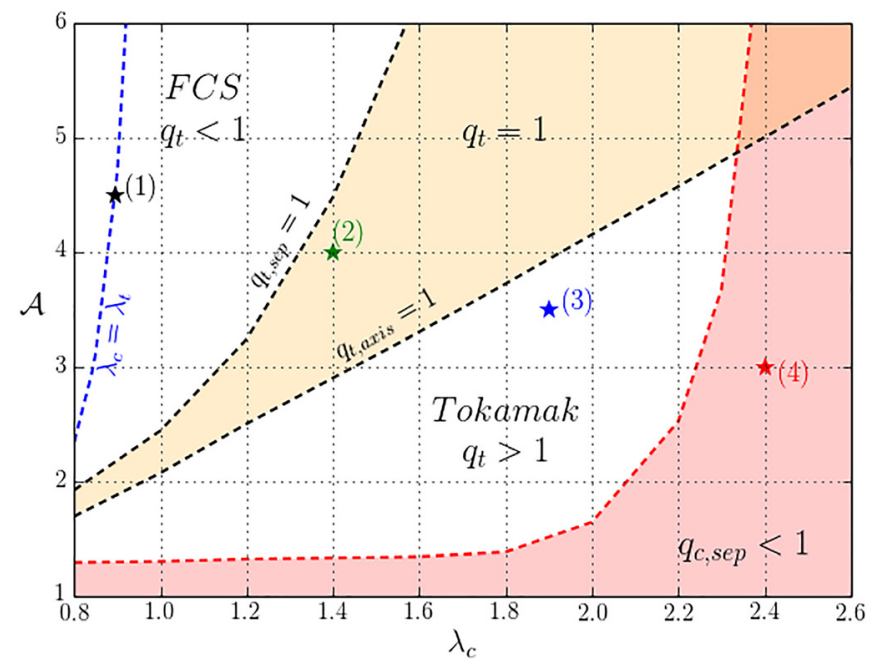

FIG. 6. Types of $q$ profiles in the parameter space. Uniform (blue line) and nearly uniform $\lambda$ profiles lead to spheromak-like $q$ profiles $\left(q_{t}<1\right)$. As $\lambda_{c}$ is increased, $q_{t}$ raises until tokamak-like $q$ profiles are obtained $\left(q_{t}>1\right)$. Typical profiles in each region (for the equilibria indicated by the stars) are shown in Fig. 5. Equilibria in the red region have $q_{c}$ below one at the separatrix.

the pinch effect caused by a high $\lambda_{c}$, as can be observed in Fig. 5.

Case (1), shown in Fig. 5, corresponds to a fully relaxed FCS configuration. Its low $\lambda_{c}$ value produces a high $q_{c}$ in the central column. In the torus, $q_{t}$ increases from the separatrix and reaches its maximum at the magnetic axis (reversed shear). This is a typical spheromak $q$ profile. ${ }^{11}$ As $\lambda_{c}$ increases and $\mathcal{A}$ decreases, the scenario changes dramatically. On the one hand, $q_{c}$ decreases significantly with $\lambda_{c}$. On the other hand, $q_{t}$ increases everywhere in the torus but most rapidly in the outer region near the separatrix. The magnetic shear changes its sign and eventually a tokamak-like $q$ profile (regular shear) is obtained with $q_{t}>1$ everywhere in the torus (cases 3 and 4).

The regions of the parameter space where configurations have spheromak-like $q$ profiles $\left(q_{t}<1\right)$ and tokamak-like $q$ profiles $\left(q_{t}>1\right)$ are indicated in Fig. 6. The intermediate region where the torus has a $q_{t}=1$ surface is shaded in yellow. The upper limit of this region is the locus of equilibria with $q_{t, s e p}=1$, i.e., with $q_{t}=1$ at the separatrix, while the lower limit is the locus of equilibria with $q_{t}=1$ at the magnetic axis $\left(q_{t, \text { axis }}=1\right)$. The stars correspond to the equilibria presented in Fig. 5.

Within the red region of Fig. 6, equilibria have $q_{c}$ at the separatrix below one $\left(q_{c, \text { sep }}<1\right)$. This condition is met at very high $\lambda_{c}$, as expected, but also at very low $\mathcal{A}$. The boundary of this region is $q_{c, s e p}=1$, which is a simple estimation of the kink stability threshold of the central column based on the Kruskal-Shafranov (KS) condition $^{16}$ (see Sec. III B).

The intersection of the regions having $q_{t}>1$ and $q_{c}>1$ (case 3) represents an attractive range of operation, since stable configurations with tokamak-like $q$ profiles can be achieved inside a simple connected chamber, as discussed in Sec. III.

\section{STABILITY AND RELAXATION}

The stability of ST configurations with high current flowing along the PCC is studied here. In the zero beta limit, only currents can drive instabilities. In this context, a sufficient condition for stability is that the configuration has a uniform $\lambda$, i.e., it is a relaxed state. ${ }^{7,18}$ However, this is not a necessary condition: there are stable configurations that have $\lambda$ gradients. It has been shown, for instance, that spheromak configurations with linear $\lambda(\psi)$ profiles are stable provided that the normalized slope is below a threshold. ${ }^{19,22,23}$

In this section, the stability of the ST-PCC configurations is investigated by solving the non-linear resistive MHD equations as described in Sec. III A. The results, including the non-linear dynamics of unstable cases leading (or not) to plasma relaxation, are presented in Sec. III B.

\section{A. Resistive MHD model}

The plasma dynamics is described using the viscoresistive MHD model. The dimensionless form of the evolution equations for the plasma velocities $\mathbf{u}$ and $\mathbf{B}$ is

$$
\begin{gathered}
\rho\left(\frac{\partial}{\partial t}+\mathbf{u} \cdot \nabla\right) \mathbf{u}=-\nabla p+\mathbf{J} \times \mathbf{B}+\frac{1}{R e} \nabla \cdot \Pi, \\
\frac{\partial \mathbf{B}}{\partial t}=\nabla \times(\mathbf{u} \times \mathbf{B})-\frac{1}{R_{m}} \nabla \times \nabla \times \mathbf{J},
\end{gathered}
$$

where $\Pi=\left(\nabla \mathbf{u}+\nabla \mathbf{u}^{T}\right)-(2 / 3) \nabla \cdot \mathbf{u}$. In addition to $a$ and $\Psi_{0}$ (see Sec. II B), we use the Alfvèn speed $c_{0}$ based on the vacuum field $B_{0}$ to normalize $\mathbf{u}$. The resulting time scale is $t_{0}=a / c_{0}$. The dimensional analysis of Eq. (12) shows that the remaining scales are related by $p_{0}=\rho_{0} c_{0}^{2}=B_{0}^{2} / \mu_{0}$. The Reynolds and magnetic Reynolds numbers are defined as usual: $R e=a c_{0} / \nu$ and $R_{m}=\mu_{0} a c_{0} / \eta$, where $\nu$ is the kinematic viscosity and $\eta$ is the plasma resistivity. The results presented below were obtained using $R e=R_{m}=5 \times 10^{4}$.

The dimensionless pressure appearing in Eq. (12) is proportional to the plasma $\beta$. As mentioned in Sec. II, the present study is focused on the zero $\beta$ limit; thus, the first term in the right hand side of Eq. (12) is neglected, and the plasma density is kept constant. With this simplification, the two unknowns are $\mathbf{B}$ and $\mathbf{u}$. The boundary condition for the normal component of $\mathbf{B}$ is obtained from the value of $\psi$ at the boundary (see Sec. II B). The tangential component of $\mathbf{J}$ must satisfy $\mathbf{J} \times \mathbf{n}=0$ in order to avoid a finite current along the boundaries. The normal component of $\mathbf{u}$ is always zero at the boundary. The tangential component is zero for the stability analysis of Sec. III B.

The system of equations is discretized and solved using standard finite volume techniques. A cartesian grid with $160 \times 160 \times 80$ volumes is employed, and the nonconforming boundary conditions are imposed using a highorder method for this type of grid. ${ }^{24}$ The hyperbolic terms are approximated by the linearized Riemann problem which is solved using the Roe method for MHD. ${ }^{25} \mathrm{~A}$ high resolution is achieved using the monotonized central-difference limiter (MC limiter). ${ }^{26}$ Central differences are employed in the discretization of the parabolic terms (viscous and resistive diffusion). The divergence free condition of the magnetic field is maintained using the projection method. ${ }^{27}$ Validation of this numerical scheme and convergence studies 
for typical spheromak conditions have already been presented. $^{12,22,23,28}$

\section{B. Results and discussion}

The stability of ST-PCC configurations is studied by solving the time-dependent MHD equations in three spatial dimensions. The initial condition for each simulation is constructed by adding a small perturbation with toroidal wave number $n=1$ to the axisymmetric $(n=0)$ magnetic field of the equilibrium. For highly unstable configurations, the dominant mode rapidly grows and can be used to construct a suitable perturbation for the other cases. ${ }^{22}$

The evolution of the magnetic energy of the $n=1$ mode, relative to the magnetic energy of the equilibrium, the amplification factor, and the toroidal flux is shown in Fig. 7 for configurations with several $\lambda_{c}$ and initial $\mathcal{A}=2.5[$ (a)-(c)] and $\mathcal{A}=4[(\mathrm{~d})-(\mathrm{f})]$.

For $\mathcal{A}=2.5$ [Fig. 7(a)], we observe that the stability threshold of the $n=1$ mode is at $\lambda_{c} \approx 2.2$, in agreement with the KS condition, expressed in terms of the safety factor as $q_{c, \text { sep }}=1$ (see Fig. 6). As expected, this mode produces the kink distortion of the central column that can be observed in Fig. 8. Simulations around the stability threshold for $\mathcal{A}=4$
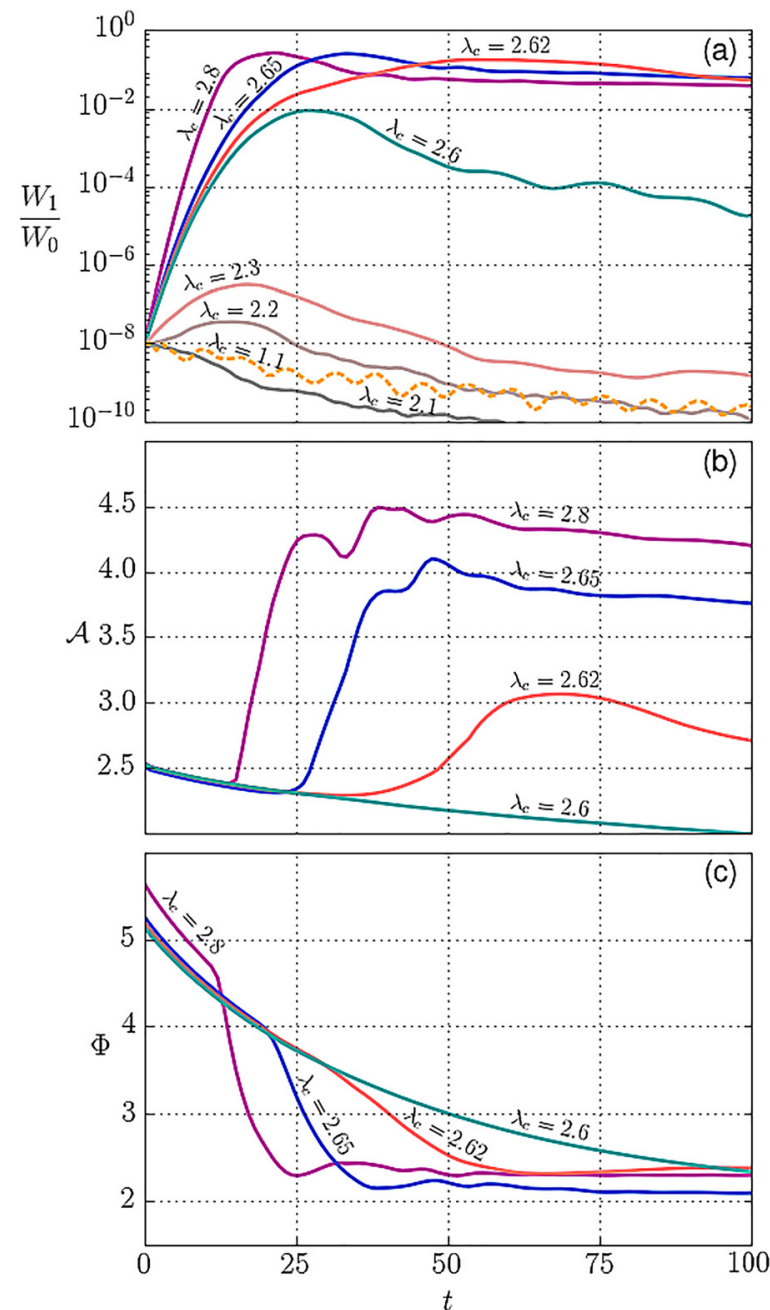

[Fig. 7(d)] are also in agreement with the KS limit. Note that in this case, the non-linear saturation phase is rapidly reached and followed by rebounds of the mode amplitude. This behavior, observed for unstable configurations up to $\lambda_{c}=2.8$, is due to the stabilizing effect of the torus which contains, for the $\mathcal{A}=4$ case, a significantly larger amount of magnetic flux.

When $\lambda_{c}$ exceeds the stability boundary, the unstable modes grow, saturate, and finally decay because there is no external driving in these simulations. It is important to note that the presence of fluctuations produced by the instability does not imply relaxation behavior. In order to observe plasma relaxation, $\lambda_{c}$ must be increased well above the stability boundary. This can be seen in Figs. 7(a) and 7(d), which shows that a very small change in $\lambda_{c}$ (from 2.60 to 2.62 for $\mathcal{A}=2.5$ and from 2.96 to 2.97 for $\mathcal{A}=4$ ) produces a very large change in the behavior of the modes at the non-linear saturation of the instability and during their final decay.

A key effect of relaxation is flux conversion, from toroidal to poloidal in this case, due to the dynamo action of the fluctuations. This effect is observed for $\lambda_{c}>2.6$, when $\mathcal{A}=2.5$, and for $\lambda_{c}>2.96$, when $\mathcal{A}=4$, as shown in Figs. 7(b) and 7(c) and 7(e) and 7(f). A remarkable aspect of these results is that the relaxation dynamics is suddenly triggered just above this threshold. Note that for $\mathcal{A}=2.5(4)$, the
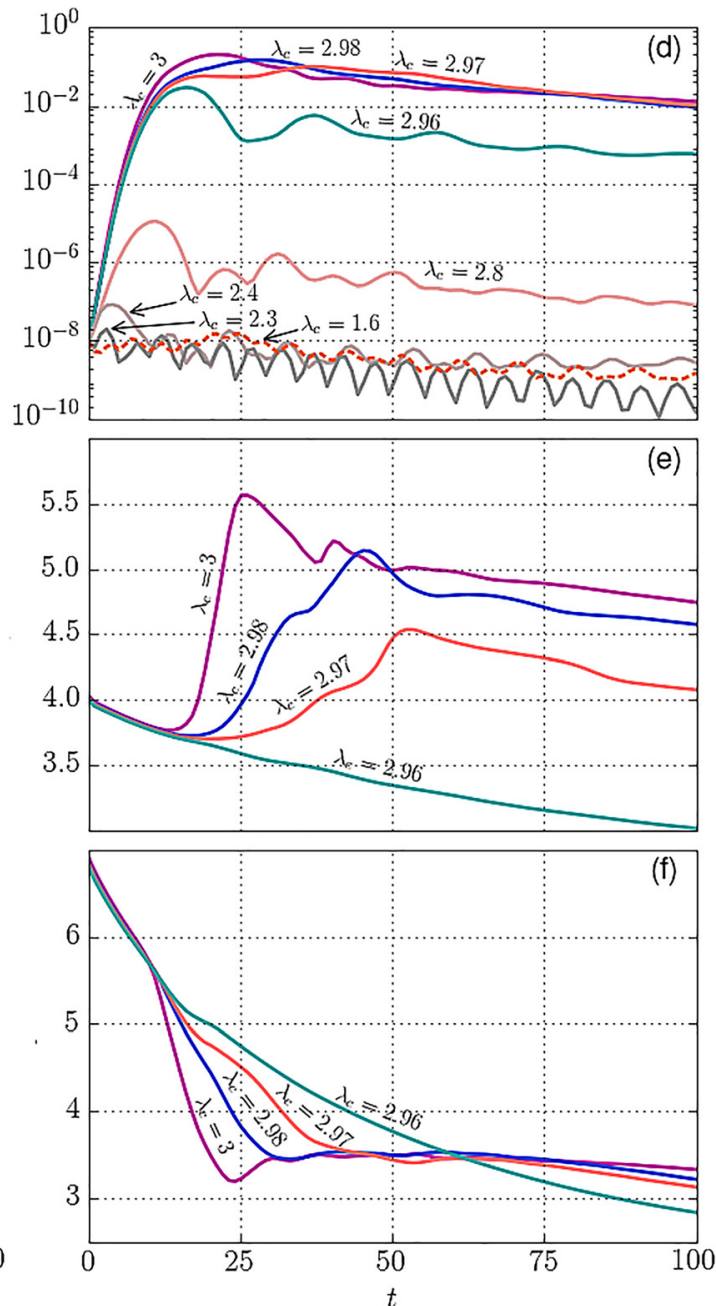

FIG. 7. Evolution of the relative magnetic energy of the $n=1$ mode $\left(W_{1} / W_{0}\right)$, the flux amplification factor $(\mathcal{A})$, and the toroidal flux $(\Phi)$ for several cases with initial $\mathcal{A}=2.5[(\mathrm{a})-(\mathrm{c})]$ and $\mathcal{A}=4[(\mathrm{~d})-(\mathrm{f})]$. 
configuration with $\lambda_{c}=2.6(2.96)$ is highly unstable but does not show any relaxation behavior, while configurations just above those values show significant flux conversion.

Another important effect of relaxation is the flattening of the $\lambda$ profile and the consequent modification of the $q$ profile. The evolution of the $\lambda(\psi)$ and $q(r)$ profiles for configurations with (initial) $\mathcal{A}=2.5$ is shown in Fig. 9. These profiles provide a measure of the degree of relaxation produced by the instability. The most unstable case $\left(\lambda_{c}=2.65\right)$ undergoes complete relaxation, and an almost flat $\lambda$ profile is rapidly achieved. Note that $q_{t}$ after relaxation is below one, as in a typical spheromak or FCS configurations. In the second case $\left(\lambda_{c}=2.62\right)$, just above the relaxation threshold, the instability produces significant flux conversion and current

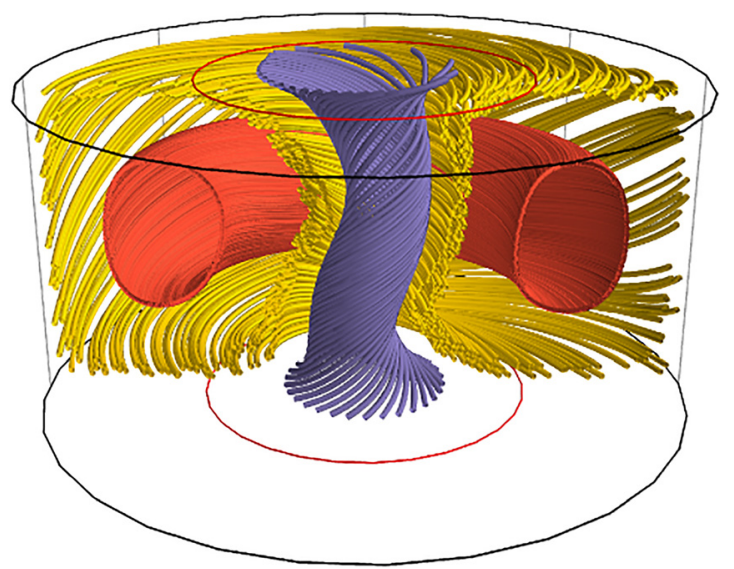

FIG. 8. Field lines showing the kink distortion of the central column and its effect on the torus, for the case with initial $\lambda_{c}=2.8$ and $\mathcal{A}=2.5$.
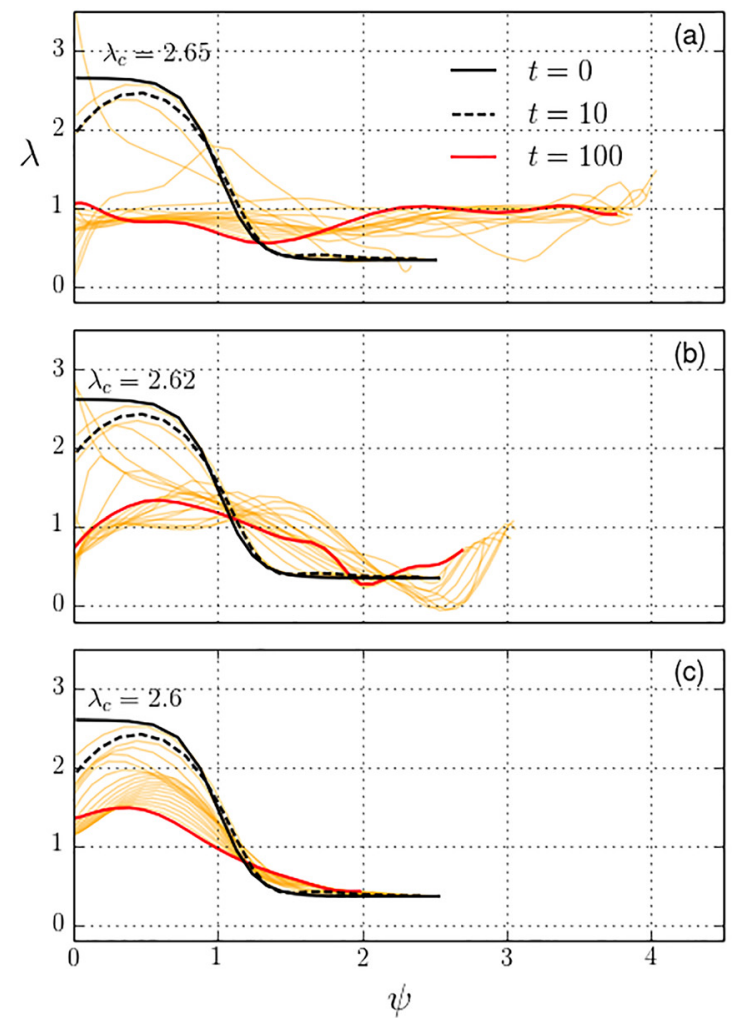

redistribution, decreasing $\lambda$ in the column and increasing $\lambda$ in the torus. However, relaxation is not complete since $\lambda(\psi)$ after the instability saturation deviates significantly from a uniform profile. Note the formation of a $\lambda$ gradient inside the torus. Finally, the evolution of the profiles of the unstable case $\lambda_{c}=2.6$ only exhibits the resistive decay of the configuration without any signature of relaxation.

As already mentioned, the stability boundary obtained is in good agreement with the KS limit for the central column. When $\lambda_{c}$ is below this limit, the amplitude of all the applied initial perturbations decayed in a similar way to that shown with dashed lines in Figs. 7(a) and 7(d). We note that these cases fall within the yellow region of Fig. 6, and thus, they involve a $q_{t}=1$ surface. In this situation, it could be expected that the torus would become unstable to a toroidal kink mode. The stable behavior obtained for these configurations is explained in terms of the lack of driving for the instability, since $\lambda$ is uniform within the torus and there are no pressure gradients. In the case of spheromaks under sustainment, for example, the external driving produces a $\lambda$ gradient, and the system becomes unstable whenever $q_{t}$ raises above one. ${ }^{12,19,22}$ The interpretation is that the rational $q_{t}=$ 1 surface triggers the instability driven by the $\lambda$ gradient (i.e., a current-driven instability). This situation also arises during sustainment by HI in ST-PCC configurations, but the $\lambda$ gradient has the opposite sign, as shown in Sec. IV.

\section{FORMATION AND SUSTAINMENT BY HELICITY INJECTION}

Magnetic helicity can be injected by applying a voltage difference between the electrodes. ${ }^{18}$ The current driven
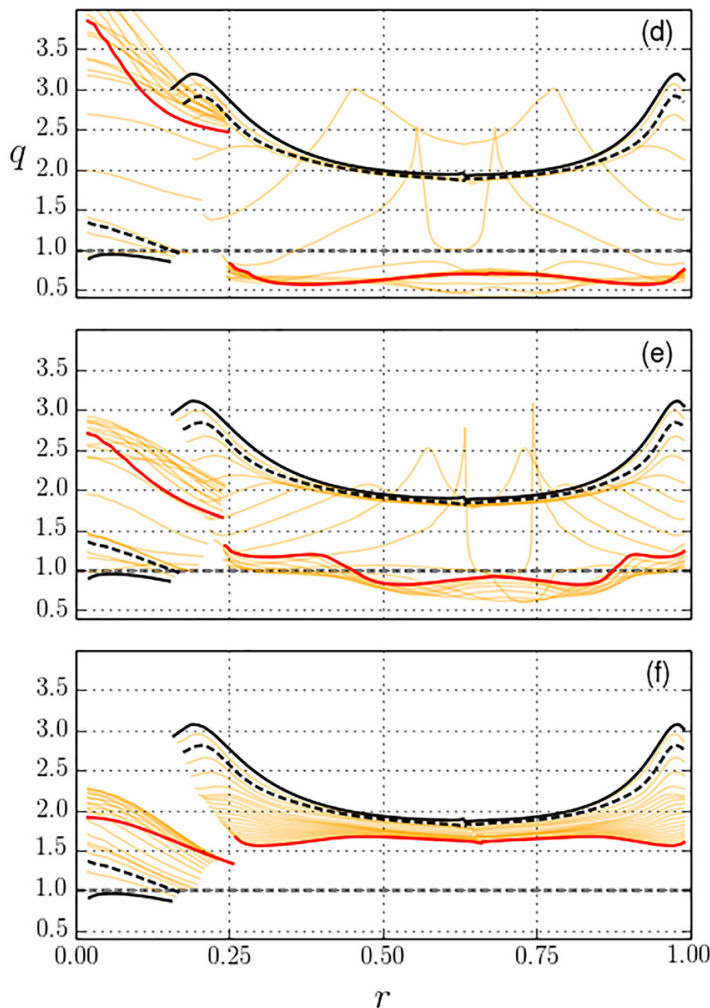

FIG. 9. $\lambda(\psi)$ profiles and radial $q$ profiles at several times for configurations with initial $\mathcal{A}=2.5$ and several $\lambda_{c}$ 

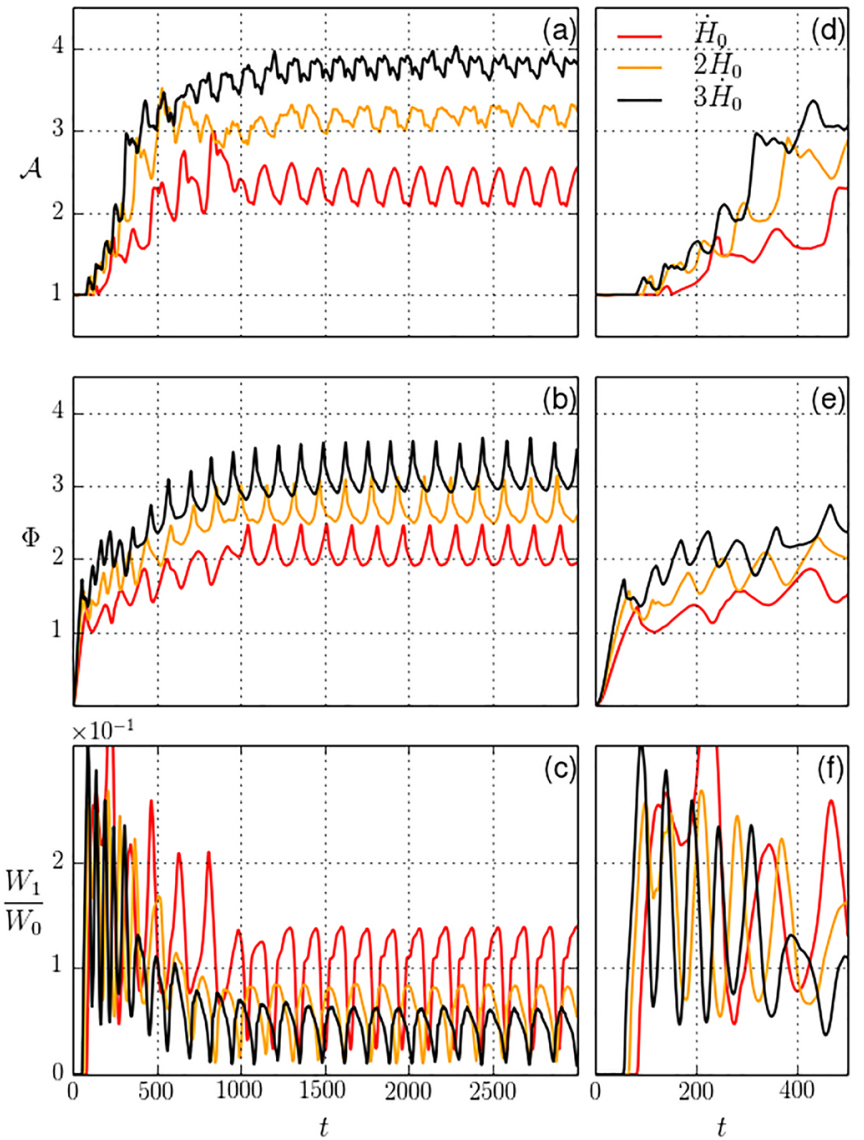

FIG. 10. Evolution of $\mathcal{A}$, the toroidal flux, and the relative amplitude of fluctuations from the vacuum field at three HI rates [(a)-(c)]. The details of the initial formation phase are shown in (d)-(f). $\dot{H}_{0}$ is the helicity injection rate obtained when the amplitude of the forcing tangential flows is set to $u_{0}=0.015$.

along the open field lines is redistributed by magnetic relaxation. This process has been successfully used to form and sustain spheromaks ${ }^{19,29,30}$ as well as low-aspect-ratio tokamaks $^{31,32}$ and other configurations. ${ }^{33}$ In this section, we present results of MHD simulations that show the feasibility of using HI to form and sustain ST-PCC configurations.

Although electrostatic potentials are not described by the MHD model, helicity can be injected by tangential flows at the electrodes. ${ }^{12,28}$ Taking the vacuum field as an initial condition, counter-rotating flows are applied at the electrodes. A simple parabolic profile is imposed for the toroidal velocity $u_{\phi}(r)=u_{0} r\left(r_{e}-r\right)$, for $r<r_{e}$ and zero otherwise, with the opposite sign at each electrode. The resulting evolution of $\mathcal{A}, \Phi$, and the relative amplitude of the fluctuations at three HI rates is shown in Fig. 10. The formation phase is shown in the right column in more detail.

The imposed toroidal velocity times the axial bias field produces a radial electric field that acts as a source of toroidal magnetic flux. As a result, a buildup of $\Phi$, proportional to the $\mathrm{HI}$ rate, is observed in the initial phase. Since there are no external sources of poloidal flux, $\mathcal{A}$ remains constant until the instabilities responsible for flux conversion are triggered, as can be observed in Figs. 10(d)-10(f).

After the formation phase, a quasi-steady-state regime is reached when the mean resistive dissipation balances the injection of helicity and energy, as can be observed in Figs. 10(a)-10(c). The sustainment of $\mathcal{A}$ implies a toroidal current driving mechanism (or dynamo) involving fluctuations. The presence of a saturated level of fluctuations implies, in turn, that the system operates at marginal stability. Figure 11 shows the $\lambda(\psi)$ and $q(r)$ profiles, for the three cases, at several times during the growth of the $n=1$ mode that precedes a poloidal flux amplification event, during the sustainment phase.

We recall that, in this case, the configuration and its $\lambda$ profile are formed spontaneously via relaxation and selforganization, from the vacuum field $(\lambda=0)$ due to the symmetric forcing imposed at the electrodes. Despite a significant level of fluctuations, the structure of the mean $\lambda(\psi)$ profiles resembles the tanh dependence adopted in Sec. II D, having a high current along the PCC. An important difference is that a $\lambda$ gradient is produced in the closed flux region. This is a result of the current redistribution produced by an incomplete relaxation event, as observed in Figs. 9(b) and 9(e). Therefore, HI sustainment occurs at marginal stability, due to the formation of a $\lambda$ gradient and a $q_{t}=1$ surface in the ST. This behavior is analogous to that observed in CHI spheromaks but shows a major difference: the $\lambda$ gradient spontaneously formed inside the torus $(\psi>1)$ has the opposite sign. Note that at the onset of poloidal flux amplification (shown in Fig. 11), $\lambda(\psi)$ is not monotonic, it has a minimum near the separatrix $(\psi \approx 1)$ and increases towards the magnetic axis. This is in contrast to the typical monotonic $\lambda$ profiles with a negative slope (decreasing towards the magnetic axis) observed in sustained spheromaks. ${ }^{12,19,28}$

As expected, sustainment using $\mathrm{HI}$ involves a significant level of fluctuations that will affect plasma confinement. Although the amplitude of the required fluctuations is known to decrease with the Lundquist number, ${ }^{12}$ it is not clear whether it will be possible to reduce them to an acceptable level. Figure 12 shows the decay of the configuration after switching off the HI. Note that the amplitude of the fluctuations rapidly decays, leading to a significant improvement of the quality of magnetic flux surfaces. Since the configurations produced in this way are expected to have good confinement properties, ${ }^{13}$ auxiliary CD systems could be applied to investigate alternative sustainment scenarios, without the MHD activity. As discussed in Secs. II F and III B, there is a considerable region of the parameter space $\left(\lambda_{c}, \mathcal{A}\right)$ where the MHD stable operation of equilibria having tokamak-like $q$ profiles is possible (see Fig. 6).

\section{SUMMARY AND CONCLUSIONS}

Several aspects of ST-PCC configurations formed inside a simply connected volume have been studied. The nonlinear MHD equilibrium problem for arbitrary current density profiles was stated in terms of the $\lambda(\psi)$ profile and solved numerically. Adopting a tanh $\lambda(\psi)$ profile, the magnetic structure and stability of the configurations were studied in the $\left(\lambda_{c}, \mathcal{A}\right)$ parameter space. According to the $q$ profile, two regions without $q=1$ surfaces were identified in this space (Fig. 6): (i) the FCS region, with $q_{t}<1$ and reversed shear ( $q$ increasing towards the magnetic axis) and (ii) the tokamak region, with $q_{t}>1$ and regular shear ( $q$ decreasing towards 

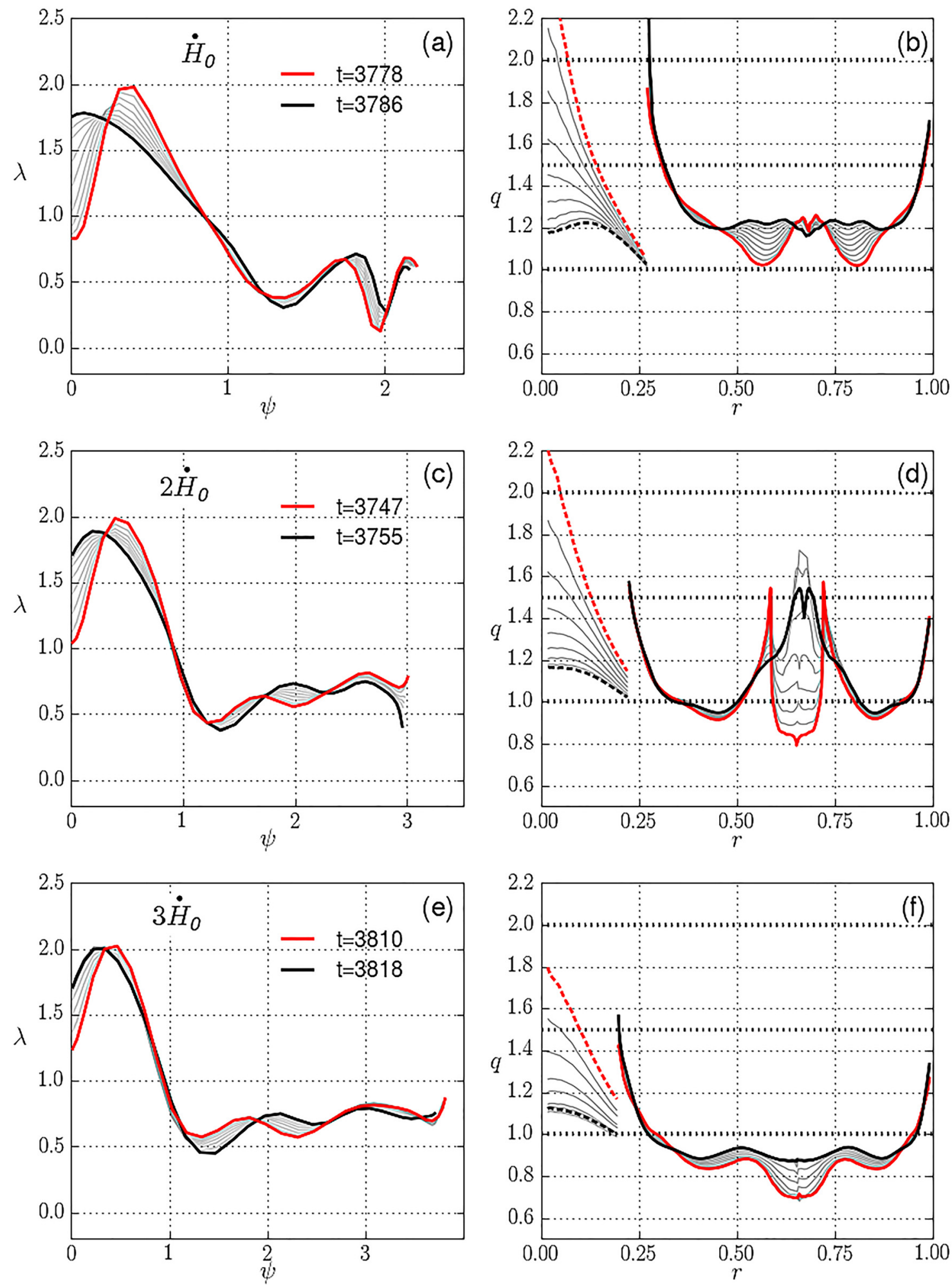

FIG. 11. $\lambda(\psi)$ and $q(r)$ profiles at the onset of poloidal flux amplification during the sustainment phase, for the three cases as shown in Fig. 10.

the magnetic axis). The former occurs for almost uniform $\lambda$ profiles and high $\mathcal{A}$, while the latter is achieved for high $\lambda_{c}$ (high current at the PCC) and moderate $\mathcal{A}(\$ 4.5)$.

The stability of these configurations was also studied. For tanh $\lambda$ profiles, a kink mode located at the PCC appears when $\lambda_{c}$ is high enough to produce a $q_{c}=1$ surface (red region in Fig. 6), which means that the PCC current is above the Kruskal-Shafranov limit. Using non-linear MHD simulations, we showed that for $\lambda_{c}$ just above this limit, the instability saturates and decays without producing current redistribution and relaxation. An unexpected result was that these processes are suddenly triggered when $\lambda_{c}$ is above a second "non-linear" threshold (which varies with $\mathcal{A}$ ). When this occurs, current is rapidly redistributed, the $\lambda$ profile is flattened, and the configuration adopts the magnetic structure of a FCS (fully relaxed configuration). This disruptive 

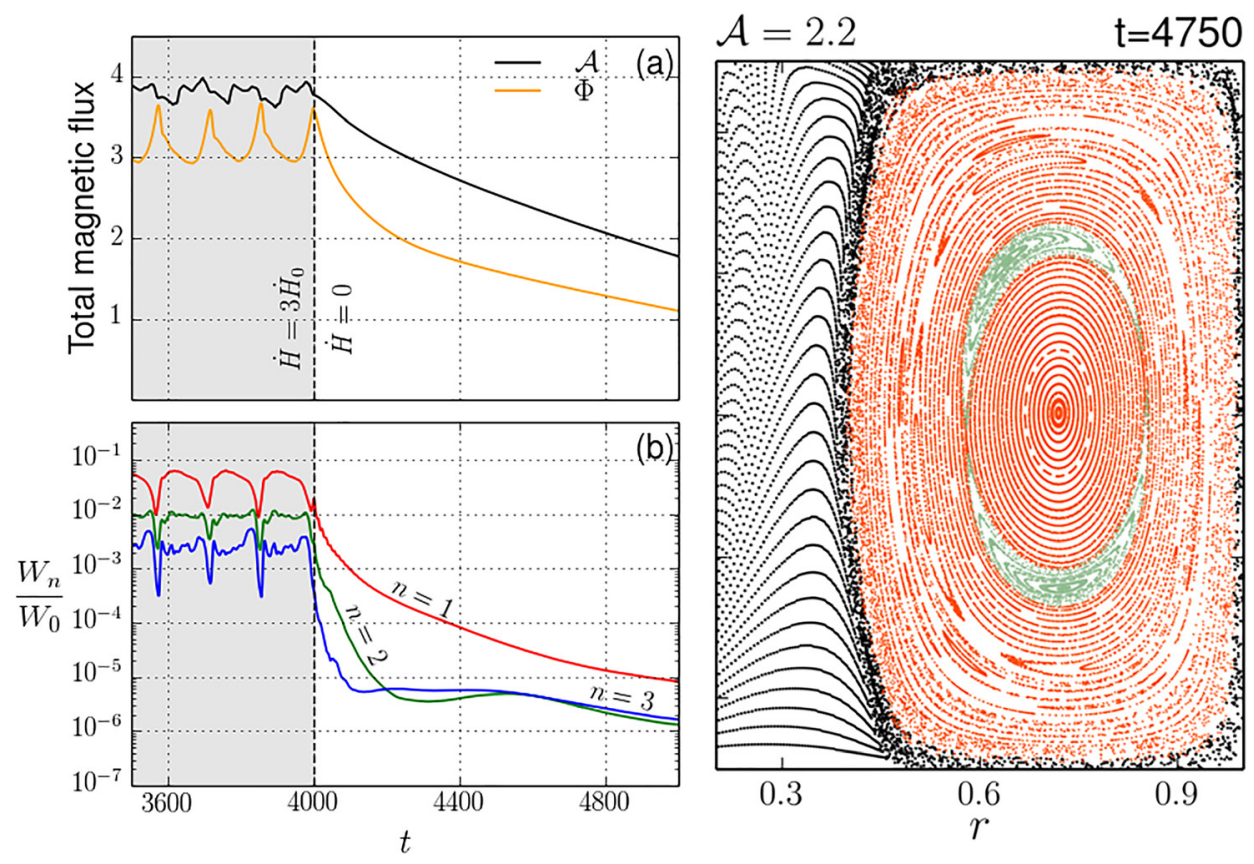

FIG. 12. Flux amplification, toroidal flux (a), and dominant fluctuations (b) decay after HI switching off. On the right, a Poincaré plot showing the regularization of field lines once the relative amplitude of fluctuations falls below $10^{-4}$. behavior is in contrast to the partial relaxation behavior observed in spheromaks with linear $\lambda$ profiles. $^{22,34}$ On the other hand, this behavior may be compared to the "trigger problem" found during sawtooth oscillations in conventional tokamaks, which involves the presence of a saturated longlasting kink mode during the last part of the ramp phase, followed by a suddenly triggered and rapid current redistribution process (i.e., the crash). ${ }^{35}$

Using counter-rotating tangential flows at the electrodes, helicity was injected to study the dynamics of formation and sustainment. This boundary condition acts as an axisymmetric source of toroidal flux that increases the current of the PCC. Eventually, the system becomes unstable, and the resulting fluctuations produce a quasi-periodic flux conversion mechanism, from toroidal to poloidal, leading to the formation and subsequent sustainment of the configuration. During sustainment, a saturated level of fluctuations is present, indicating that the system operates at marginal stability. In analogy with spheromaks sustained by a coaxial gun, ${ }^{12,19,28}$ this marginally stable configuration has a $\lambda$ gradient and a $q_{t}=1$ surface in the ST. However, two major differences were found for ST-PCC configurations. First, the $\lambda(\psi)$ profile has a very high $\lambda_{c}$, indicating a higher current density along the PCC, and second, the $\lambda$ gradient in the ST has the opposite sign, leading to tokamak-like $q$ profiles. To the best of our knowledge, the spontaneous formation and sustainment using HI of this type of profile in compact configurations have not been reported before.

Although the behavior of fluctuations for different conditions, such as different Lundquist numbers, was not studied, these results suggest that the level of fluctuations during sustainment by HI through the PCC may be higher than in coaxial HI sustainment of spheromaks. ${ }^{12}$ This motivates future investigations of more complex schemes, combining PCC and coaxial HI for instance, to explore the limits of the HI sustainment scenario. In addition, the results of this work also encourage a more detailed study of the use of auxiliary
CD combined with the expected large bootstrap current for an alternative sustainment scenario without the MHD activity. Moreover, our results indicate that good quality flux surfaces can be obtained shortly after the HI is switched off. Using this configuration as the initial target for the auxiliary $\mathrm{CD}$ systems, the stable region with the tokamak-like $q$ profile, in the parameter space $\left(\lambda_{c}, \mathcal{A}\right)$, could be explored.

Although many new and interesting results have been obtained in this study, additional studies are needed to assess the full potential of ST-PCC configurations. First, our physical model can be improved by adding finite pressure effects. This is important for configurations that are expected to operate at high $\beta$ values. The addition of finite pressure, and pressure gradients, could modify the stability boundaries and the evolution of the modes. Second, the effect of changing the elongation and the shape of the boundary should be studied. It is known that STs are naturally elongated, and this contributes to increasing the value of the safety factor. Finally, shaping the boundary as is done in the PROTOSPHERA experiment ${ }^{4}$ could improve the stability of the configuration.

\section{ACKNOWLEDGMENTS}

Financial support from UNCuyo Project No. 06/c494 is acknowledged.

${ }^{1}$ Y.-K. Peng and D. Strickler, Nucl. Fusion 26, 769 (1986).

${ }^{2}$ S. Gerhardt, D. Gates, S. Kaye, R. Maingi, J. Menard, S. Sabbagh,

V. Soukhanovskii, M. Bell, R. Bell, J. Canik, E. Fredrickson, R. Kaita,

E. Kolemen, H. Kugel, B. L. Blanc, D. Mastrovito, D. Mueller, and

H. Yuh, Nucl. Fusion 51, 073031 (2011).

${ }^{3}$ M. Ono and R. Kaita, Phys. Plasmas 22, 040501 (2015).

${ }^{4}$ F. Alladio, P. Costa, A. Mancuso, P. Micozzi, S. Papastergiou, and F. Rogier, Nucl. Fusion 46, S613 (2006).

${ }^{5}$ X. Z. Tang and A. H. Boozer, Phys. Plasmas 13, 42514 (2006).

${ }^{6}$ A. Lampasi, G. Maffia, F. Alladio, L. Boncagni, F. Causa, E. Giovannozzi,

L. A. Grosso, A. Mancuso, P. Micozzi, V. Piergotti, G. Rocchi, A. Sibio,

B. Tilia, and V. Zanza, Energies 9, 508 (2016).

${ }^{7}$ J. B. Taylor, Rev. Mod. Phys. 58, 741 (1986). 
${ }^{8}$ S. C. Hsu and X. Z. Tang, J. Fusion Energy 26, 85 (2007).

${ }^{9}$ L. G. Lampugnani, P. L. Garcia-Martinez, and R. Farengo, Phys. Plasmas 24, 022501 (2017)

${ }^{10}$ T. R. Jarboe, Plasma Phys. Controlled Fusion 36, 945 (1994).

${ }^{11} \mathrm{P}$. M. Bellan, Spheromaks: A Practical Application of Magnetohydrodynamic Dynamos and Plasma Self-Organization (Imperial College Press, London, 2000).

${ }^{12}$ P. L. García Martínez, L. G. Lampugnani, and R. Farengo, Phys. Plasmas 21, 122511 (2014).

${ }^{13}$ F. J. Wysocki, J. C. Fernndez, I. Henins, T. R. Jarboe, and G. J. Marklin, Phys. Rev. Lett 65, 40 (1990).

${ }^{14}$ J. S. Sarff, S. A. Hokin, H. Ji, S. C. Prager, and C. R. Sovinec, Phys. Rev. Lett. 72, 3670 (1994).

${ }^{15}$ E. B. Hooper, Plasma Phys. Controlled Fusion 53, 085008 (2011).

${ }^{16}$ J. P. Freidberg, Ideal MHD (Cambridge University Press, 2014).

${ }^{17}$ D. A. Kitson and P. K. Browning, Plasma Phys. Controlled Fusion 32, 1265 (1990).

${ }^{18}$ T. H. Jensen and M. S. Chu, Phys. Fluids 27, 2881 (1984).

${ }^{19}$ S. O. Knox, C. W. Barnes, G. J. Marklin, T. R. Jarboe, I. Henins, H. W. Hoida, and B. L. Wright, Phys. Rev. Lett. 56, 842 (1986).

${ }^{20}$ D. M. Willett, P. K. Browning, S. Woodruff, and K. J. Gibson, Plasma Phys. Controlled Fusion 41, 595 (1999).

${ }^{21}$ T. Jarboe, B. Victor, B. Nelson, C. Hansen, C. Akcay, D. Ennis, N. Hicks, A. Hossack, G. Marklin, and R. Smith, Nucl. Fusion 52, 083017 (2012).
${ }^{22}$ P. L. García Martínez and R. Farengo, Phys. Plasmas 16, 082507 (2009).

${ }^{23} \mathrm{P}$. L. Garcia-Martinez, in Topics in Magnetohydrodynamics, edited by L. Zheng (InTech, 2012), Chap. 4, pp. 85-116.

${ }^{24}$ H. Forrer and R. Jeltsch, J. Comput. Phys. 140, 259 (1998).

${ }^{25}$ P. L. Roe and D. S. Balsara, SIAM J. Appl. Math. 56, 57 (1996).

${ }^{26}$ B. van Leer, J. Comput. Phys. 23, 276 (1977).

${ }^{27}$ G. Toth, J. Comput. Phys. 161, 605 (2000).

${ }^{28}$ P. L. García Martínez and R. Farengo, Phys. Plasmas 17, 050701 (2010).

${ }^{29}$ M. G. Rusbridge, S. J. Gee, P. K. Browning, G. Cunningham, R. C. Duck, A. al-Karkhy, R. Martin, and J. W. Bradley, Plasma Phys. Controlled Fusion 39, 683 (1997).

${ }^{30}$ E. B. Hooper, R. H. Bulmer, B. I. Cohen, D. N. Hill, H. S. McLean, L. D. Pearlstein, C. A. Romero-Talams, C. R. Sovinec, B. W. Stallard, R. D. Wood, and S. Woodruff, Plasma Phys. Controlled Fusion 54, 113001 (2012)

${ }^{31}$ B. A. Nelson, T. R. Jarboe, D. J. Orvis, L. A. McCullough, J. Xie, C. Zhang, and L. Zhou, Phys. Rev. Lett. 72, 3666 (1994).

${ }^{32}$ R. Raman, B. A. Nelson, M. G. Bell, T. R. Jarboe, D. Mueller, T. Bigelow, B. LeBlanc, R. Maqueda, J. Menard, M. Ono, and R. Wilson, Phys. Rev. Lett. 97, 175002 (2006).

${ }^{33}$ S. C. Hsu and P. M. Bellan, Phys. Plasmas 12, 032103 (2005).

${ }^{34}$ P. L. García Martínez and R. Farengo, J. Phys. Conf. Ser. 166, 012010 (2009).

${ }^{35}$ R. J. Hastie, Astrophys. Space Sci. 256, 177 (1997). 